\title{
Radio Stations and Audience Communication: Social Media Utilization and Listeners Interaction
}

\author{
Zacharenia Pilitsidou \\ Aristotle University of Thessaloniki, Greece \\ E-mail: zpilitsidou@gmail.com \\ Nikolaos Tsigilis \\ Aristotle University of Thessaloniki, Greece
}

George Kalliris

Aristotle University of Thessaloniki, Greece

Received: May 4, 2019

Accepted: May 31, 2019

Published: June 8, 2019

doi:10.5296/iss.v7i1.14743

URL: http://dx.doi.org/10.5296/iss.v7i1.14743

\begin{abstract}
The purpose of the study was to explore how Greek radio stations utilize social media and Facebook in particular, and to look into factors that influence interaction with their audience. Twenty radio stations broadcasting from the two largest cities in Greece were selected according to their radio profile and number of followers. The duration of the study was two weeks, weekends included, with one month time lag between them. Results showed that the type of content seems to influence audience's participation. Listeners had higher participation when they were reading information with content of their favourite radio broadcasting. Moreover, it was noticed that a specific time of the day might facilitate communication between radio stations and their listeners. Interaction patterns differ in relation to radio profile and radio stations broadcasting location. This paper represents a first attempt to investigate the ways Greek radio stations utilize social media to accomplish higher participation levels. An interaction index was introduced and used in order to better reflect radio station audience interaction. Given the relatively short observation period present these findings should be considered preliminary and exploratory. Longer data collection period combined with alternative social media such as Twitter, can provide a deeper understanding of the topic. Radio stations can utilize the present findings to develop a more effective strategy
\end{abstract}


communication through social media.

Keywords: Social media, Communication, Interaction, Radio stations 


\section{Introduction}

Nowadays, an increasing number of citizens become active users of social media and use them as their main communication channel in their daily lives. The second quarter of 2018 , Facebook had 2.23 billion monthly active users, which makes it the most popular social network worldwide (www.statista.com). Also, media of social networking are being used by more and more radio stations as a means of promoting their programs to increase their ratings. They have become the most preferred form of online communication with and among listeners, additionally new communication technologies do not only improve the relationship between the audiences and the radio at just quantitative context but also affect the closeness of qualification to connect audiences to radio (Tufan, 2014). Thus, there is a continuously increasing interaction between the radio industry and its audience.

As social media prevailed people's everyday life, various questions were raised regarding their use by radio stations. For example, researchers examined how audiences use radio stations' Facebook page, and the interaction between the radio presenter and audiences. They concluded that computer, internet and communication technologies such as social networking sites offered audience a more active identity and helped the radio world achieve tremendous developments (Emine \& Guzin, 2011). The differences between advertising, public and communal radio stations were studied in Canada (Bonin, 2016). Bonin (2016) used a face to face interview of four managers and thirteen journalists to fully comprehend the reasons social media are being used. In another study Bonini (2014) attempted to answer the current issue of the changes that radio has undergone since it started to operate alongside social media. Among the various existing social media he specifically focused on Facebook. Finally, the extent to which radio broadcasters influence the degree of off-air contact or interaction between music radio stations and their listeners was studied in Germany. The results showed that the bond between a radio station and its listeners is not only determined by the frequency and duration of the listeners tuning into the station, but also by the number of listeners active participation off air (Spangardt, Ruth, \& Schramm, 2016).

Several studies examined how radio stations incorporate social media in everyday broadcasting as well as radio stations strategy to develop and maintain an online community. Results from various countries and cultural backgrounds seem to suggest that radio stations do not have a specific strategy and they rather experiment. For instance, a study by Freeman, Klapczynsk and Wood (2012) focused on the use of Facebook at 57 radio stations in the USA, Germany and Singapore, aiming to understand how social media were being used to create an online community. They concluded that there were no similar rules for Facebook posting, which they were covering any topic at any time. A case study of ten European researchers from seven countries on a sample of 28 public radio stations analyzed how the social media have been used for public service, and they found out that there was a common feature among all the programs. "The most engaging programs were those who invested time and human resources into nurturing the relationship with their listeners through social media" (Bonini et al., 2014, p. 103). It was becoming apparent by radio producers that certain strategies could be developed by means of the social media, especially Facebook. Posting daily on Facebook, could promote and advertise their program at specific times, interact with 
the audience and ask them to participate. In Italy these strategies were recorded and a guide was created for radio producers on how to make the most of social media before and during a radio show (Bonini, 2014).

As time went by, the use of social media kept increasing and audiences were getting more and more actively engaged in the daily flow of a radio show. Al-Rawi (2016) analyzed almost 184.000 comments to study the interaction between two popular Arabic speaking radio stations in France. He concluded that any observed interaction was indeed the result of the radio presenters asking questions and drawing the listener's attention to their topic of discussion. In Spain one of the longest researches in duration (from 2004 to 2014) took place, with the aim to record the changes in terms of audience interaction since Facebook first appeared. The National Radio of Spain was the main focus of the study, moreover it was noticed that radio in order to stay in touch with audience will slowly adopt the technology (Martín, 2016).

In the above mentioned studies it was revealed that the majority of the radio stations used Facebook as the main social medium, because users can engage with multiple platforms as diverse sources of social capital at a given time, but Facebook was found to be the dominant one (Zelenkauskaite \& Sim, 2015). This is not a peculiar observation since there is a tendency for researchers to focus on one of the various existing social media. In Italy (Bonini, Caliandro, \& Massarelli, 2015) explored the properties of the networked publics connected to radio and the audience in one platform such as Twitter. Audience interactions with radio stations was assessed mainly on counting Facebook likes. However, solely relying on Facebook likes does not capture the full range of listeners reactions, since it ignores important aspects of communication such as hearts, emotions, shares, etc. To the best of our knowledge only one study used a more informative index of audience interaction by incorporating likes, shares and comments (Pletikosa \& Michahelles, 2013). Despite their valuable effort their index did not include emotional reactions (e.g. hearts, emotions) because they were not introduced at the time of the study. It is rational to assume that an index incorporating all the available Facebook tools for communication might better reflect audience interaction with the radio stations.

The purpose of the present study was to investigate possible factors that influence the interaction between Facebook pages of the radio stations and their followers in the Greek setting. Toward this end it would be useful to explore which post the audience likes the most and which are the ones that bring to the higher participation levels.

In particular, we are interested in exploring how radio stations utilize social media as well as how their listeners-users respond to the page administrators' posts. We feel that this endeavor has both theoretical and practical merit. If we understand what is happening in Greece, where there have been few researches, we will have a representation in a different cultural domain. On the other hand, it will give to the radio station empirical data for planning the most effective use of social media. Three research questions were postulated and subsequently examined.

(a) Which type of post content attracts the audience attention and its subsequent reaction? 
(b) Does the time of the day influence the intensity of interaction?

(c) Does the interaction between radio stations and listeners differ across broadcasting location (e.g. capital and second largest city) and the profile of each station (e.g. news or entertainment)?

\section{Method}

\subsection{Radio Stations Selection}

In the present study twenty radio stations from the two largest cities in Greece (Athens and Thessaloniki) were selected and studied. The list of the radio stations was obtained from the www.greekradios.gr. The criteria for selecting a radio station were the following: (a) broadcasting from the two largest cities in Greece, (b) maintain a Facebook page, and (c) the Facebook page to be active at least for the last twenty-four months. At the next stage of selection, ratings and presence of radio stations in social media were taken into consideration. In particular, radio stations with the highest and lowest activity in Facebook were chosen in order to highlight their differences. Selection process resulted in ten radio stations broadcasting from Athens and the rest from Thessaloniki. Based on radio stations' web profile, five were mainly news stations and fifteen entertainment-music stations.

\subsection{Collection of Empirical Data}

The duration of the study was two weeks, with one month time lag between them. Each radio station was followed for 24 hours a day, seven days a week. The following posts' characteristics to Facebook radio pages were recorded: (a) number of likes of each post, (b) time of the day in which each post was uploaded, (c) content of the post (d) number of shares of the posts, (e) number of comments and (f) number of emotions. Based on prior research the content of the posts classified into information-news, promotion, and entertainment-music (Mislow, 2015; Pletikosa et al., 2013) by the principle author of the study. The time of the day was categorized into four levels, namely "Morning", which includes posts that were posted between 6.00 to 11.59 , the next category is "Afternoon" 12.00 to 17.59 , the category "Night" is between the hours 18.00 to 23.59, and the last one is "Midnight", which includes all the posts between 00 to 6.00 (Zudrell, 2016). All the above characteristics were recorded at least 24 hours after the post publication, so that social media users had enough time to respond (Freeman et al., 2012).

Audience interaction with radio stations was measured using an interaction index (Pletikosa et al., 2013). This index represents the ratio of the sum all types of listeners' responses to radio station posts (number of likes, shares, comments and emotions) over the number of followers of each radio station at the specific day. An interaction index of 1 denotes that the number of audience reactions equals the number of Facebook followers.

\section{Results}

\subsection{Content of Posts}

One-way ANOVA test was employed to examine whether audience interaction with radio 


\section{Al Macrothink}

stations as captured by the interaction index differed across the three contents of post, namely news, music, and promotion. Initial examination of the data showed significant departure from the normal distribution and thus a log transformation was used. In this analysis the normal distribution assumption was met but not the equality of variances. Hence the Welch robust test was used. Results showed a significant effect, $F(2,894.09)=90.69, p<.001$. Post hoc analysis using Games-Howell criterion revealed that promotion content yielded higher values in comparison to music and news content $(p<.001)$, and news content produced more interaction than news content $(p<.001)$ (Figure 1). In table 1 are presented the interaction index values after the log transformation.

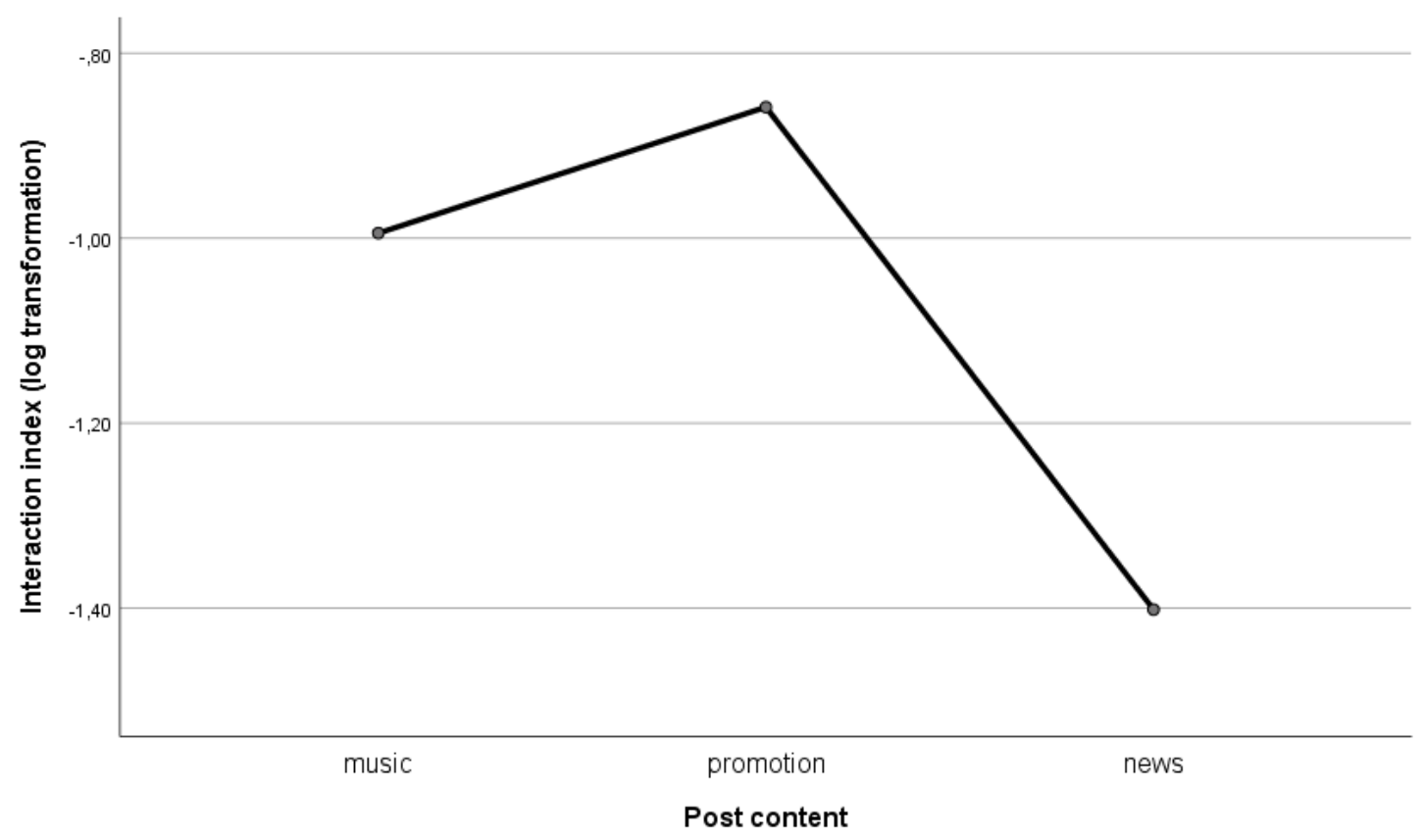

Figure 1. Differences in interaction index across the three types of post content

\subsection{Time of Post}

Initial examination of the data showed that the 0-6h time-period had substantially low observations in comparison to the other three periods (Table 1). Thus, it was decided to exclude the 0-6h time-period from the analysis. One-way ANOVA showed no statistically significant differences among the remaining three time-periods, $F(2,1821)=.535, p=.585$.

\subsection{Radio Location and Radio Type}

Two-way ANOVA test was used to examine whether the location of the radio station and its type (news or entertainment) influences audience interactions. Findings showed that both main effects were significant, $F(1,1862)=54.28, p<.001$ for location and $F(1,1862)=9.58$, $p=.002$ for radio station type. Moreover, the interaction among the two independent variables also reached statistical significance, $F(1,1862)=9.01, p=.003$. Simple main effects analysis was employed to further explore and understand the interaction (Figure 2). Results revealed that the Thessaloniki radio stations elicited more reactions in comparison to 


\section{Macrothink}

Issues in Social Science

ISSN 2329-521X 2019, Vol. 7, No. 1

the Athens radio stations ( $p=.017$ and $p<.001$ for news and entertainment respectively). The type of radio station seemed not to affect audience interaction in Athens. On the other hand, the Thessaloniki audience showed greater more reactions to the entertainment stations than to the news ones $(p<.001)$ (Table 1).

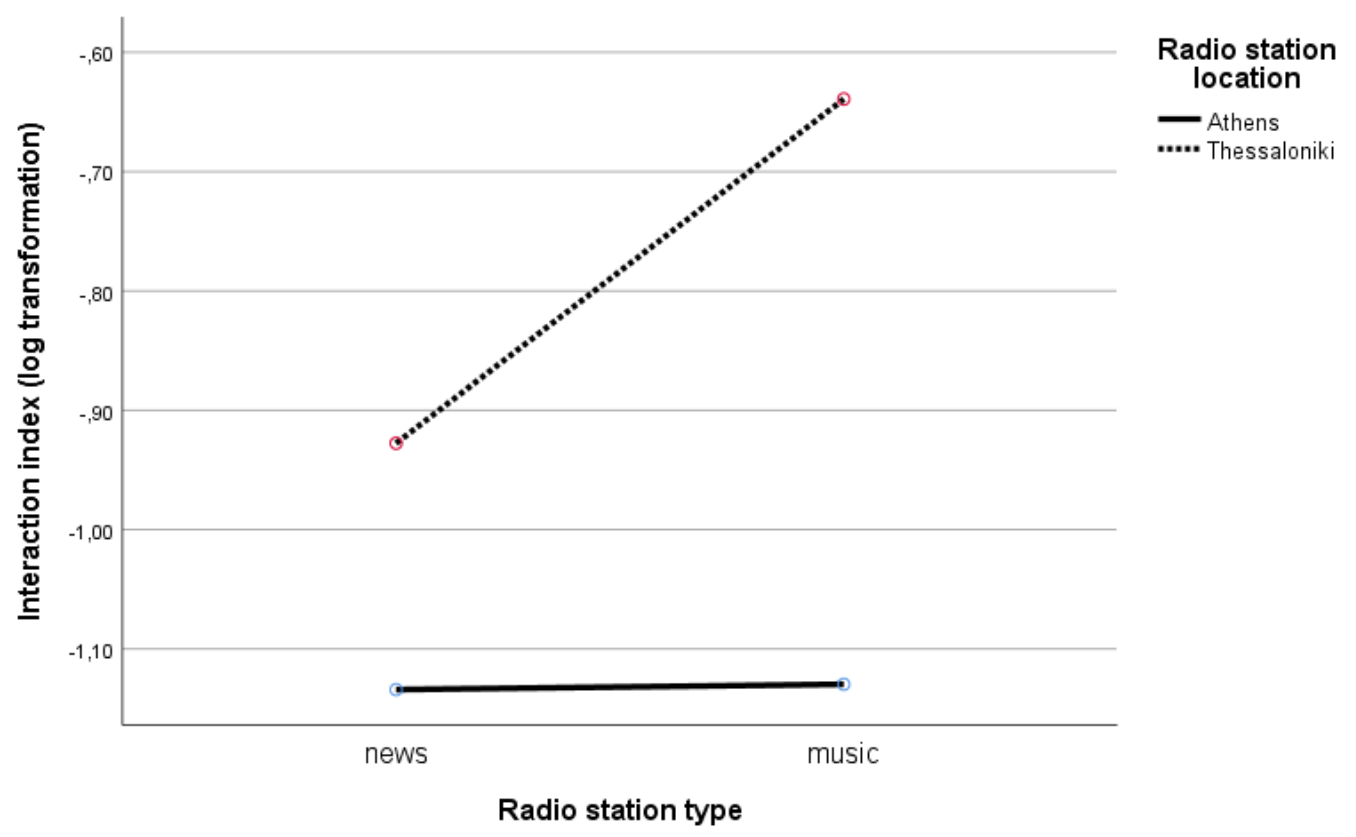

Figure 2. Differences interaction index across type of radio station and its location

Table 1. Descriptive statistics of the interaction index across the examined independent variables

\begin{tabular}{lccc}
\hline & $\mathrm{N}$ & Mean (SD) & $\begin{array}{c}\text { Log } \\
\text { Mean (SD) }\end{array}$ \\
\hline $\begin{array}{l}\text { Post content } \\
\text { Music }\end{array}$ & 425 & $.207(.34)$ & $-.995(.54)$ \\
Promotion & 1066 & $.474(.86)$ & $-.858(.76)$ \\
News & 367 & $.133(.49)$ & $-1.402(.64)$ \\
Post time & & & \\
$0-6 \mathrm{~h}$ & 42 & $.260(.25)$ & $-.800(.48)$ \\
6-12h & 471 & $.293(.55)$ & $-1.012(.69)$ \\
12-18h & 760 & $.359(.75)$ & $-.982(.72)$ \\
18-24h & 594 & $.374(.82)$ & $-1.021(.76)$ \\
Athens & & & \\
News & 139 & $.236(.75)$ & $-1.134(.66)$ \\
Music & 1156 & $.315(.71)$ & $-1.129(.76)$ \\
Thessaloniki & & & \\
News & 118 & $.298(.56)$ & $-.928(.55)$ \\
Music & 453 & $.466(.76)$ & $-.639(.54)$ \\
\hline
\end{tabular}




\section{Discussion}

The objective of this paper was to contribute to a better understanding of the way audience react to radio stations Facebook posts. This study used a more informative index of listeners' reactions to radio station posts and it was realized in the Greek cultural context, which is rarely studied. To this end, findings can be useful to radio stations presenters in order to attract more listeners and simultaneously increase participation levels.

The first research question was set to investigate the popularity of the radio stations' post content. Findings showed that posts falling in the promotion category elicited most of the audience reactions. Thus, advertising and promoting radio program or radio producers through social media might be an effective strategy to engage listeners to participate and actively involved to everyday radio broadcasting. The second most popular radio post content was entertainment, followed by news. It is interesting that news was the least popular to the audience. Our findings are not in line with those of Pletikosa et al. (2013), in which posts with entertainment content attracted audience participation in the industry setting. It seems that social media followers do not interact similarly to posts contents but they fluctuate in relation to the enterprise (e.g. radio stations, industry). The audience liked promotion content most because of their relationship with the radio presenters. According to Spangardt et al. (2016), radio personalities are important to music radio stations as they influence how and to what extent the listeners want to interact. On the other hand, it seems that radio stations listeners prefer to simply read the news and not interact or engage in a discussion (Pletikosa et al., 2013).

It is well known that radio station rating fluctuates during the day. The second research question focused on exploring which time of the day attracted listeners' participation. Results indicated that there were no differences among the three examined time periods. Similar findings were reported by Pletikosa et al. (2013) in the field of brands setting. Despite however the non-statistical significance of the results, it is worth noting that the $18-24 \mathrm{~h}$ time period yielded the higher reaction. That time of the day most of the listeners do not have obligations and can interact mostly with to radio station posts. Apart from the scientific merit, this question has also practical implications for people working in radio stations and use the social media. If the purpose is to produce more interaction and engage listeners the time period from 18 to 24 might be the most appropriate.

The third research question focused on examining the difference of the interaction in the profiles of the radio stations and whether the diversity expanse across broadcasting location. Results displayed that there is neither a difference in the interaction of news radio stations nor a difference between the examined cities. On the contrary, there is an obvious discrepancy in the radio stations with entertainment content, where the listeners of Thessaloniki showed higher engagement than the ones in Athens. This is not a coincidence because there is a long-lasting relationship of trust on the radio stations of Greece's second city. More specifically, in a period that media where mainly dominated by state radio stations, Thessaloniki pioneered in creation and maintenance private communal and regional radio stations (Theodosiadou, 2008). In addition although, the population of Athens is almost four 
times bigger than Thessaloniki's they broadcast almost the same number of radio stations. Thus, the ratio of radio station to audience is substantially larger in Thessaloniki.

Of course this study has its limitations. These limitations should be stated to help researchers working in relevant areas in the near future. The time period during which the study was conducted is rather short. Thus, longer observation periods could provide a deeper understanding between radio stations and audience interaction. It would be also interesting to explore whether listeners communication behavior differs among various seasons, in which their activities change, for instance during summer and winter. The present study was focused on Facebook, which is currently the most widely spread social medium. However, examination of other social media might reveal different audience behavior ( Bonini \& Sellas, 2014). Hence, we feel that is necessary to expand the present study in another social medium such as Twitter, to understand the similarities or differences among various communication channels.

While the relationship between radio and Facebook is still evolving, some useful conclusions about everyday posting are necessary. The type of post seems to matter, in particular because listeners are more interested in information's about the radio producers and their broadcasting, in comparison to entertainment or news posts. The time of the day does not influence the participation, although there is a particular time period that distinguish. Finally, the culture of the radio stations broadcasting location, combined with radio stations' (web) profile, seems to differentiate the listener's interactivity.

\section{References}

Al-Rawi, A. (2016). Understanding the social media audiences of radio stations. Journal of Radio and Audio Media, 23(1), 50-67. https://doi.org/10.1080/19376529.2016.1155298

Bonin, G. (2016). Organizational factors influencing journalists use of user-generated content : A case of Canadian radio newsrooms. The Radio Journal - International Studies in Broadcast \& Audio Media, 14(1), 109-122. https://doi.org/10.1386/rjao.14.1.109_1

Bonini, T. (2014). Doing radio in the age of Facebook. Radio Journal, 12(1-2), 73-87. https://doi.org/10.1386/rjao.12.1-2.73-1.

Bonini, T. et al. (2014). Radio formats and social media use in Europe: 28 case studies of public service practice. The Radio Journal - International Studies in Broadcast \& Audio Media, 12(1-2), 89-107. https://doi.org/10.1386/rjao.12.1-2.89.

Bonini, T., \& Sellas, T. (2014). Twitter as a public service medium? A content analysis of the Twitter use made by Radio RAI and RNE. Communication \& Society, 27(2), 125-146.

Bonini, T., Caliandro, A., \& Massarelli, A. (2015). Understanding the value of networked publics in radio: Employing digital methods and social network analysis to understand the Twitter publics of two Italian national radio stations. Information, Communication \& Society, 19(1), 1-28. https://doi.org/10.1080/1369118X.2015.1093532

Emine, O. A., \& Guzin, K. (2011). Mutual interaction of radio world and fan pages on 


\section{Macrothink}

Facebook. The International Journal of the Humanities, 8(10), 1-21. https://doi.org/10.18848/1447-9508/CGP/v08i10/43039

Freeman, B. C., Klapczynski, J., \& Wood, E. (2012). Radio and Facebook: The relationship between broadcast and social media software in the U.S., Germany, and Singapore. First Monday, 17(4), 1-12. https://doi.org/10.5210/fm.v17i4.3768.

Martín, M. M. (2016). How social media has affected audience 's direct participation in Spanish radio stations. The case of the National Radio of Spain. Radio, Sound \& Society, 1(1), 90-99.

Mislow, A. (2015). New radio technology and future trends. Aristotle University. Unpublished Master Thesis.

Pletikosa, C. I., \& Michahelles, F. (2013). Online engagement factors on Facebook brand pages. Social Network Analysis and Mining, 3(4), 843-861. https://doi.org/10.1007/s13278-013-0098-8.

Spangardt, B., Ruth, N., \& Schramm, H. (2016). ... And please visit our Facebook page, too! How radio presenter personalities influence listeners interactions with radio stations. Journal of Radio and Audio Media, 23(1), 1-28. https://doi.org/10.1080/19376529.2016.1155710.

Theodosiadou, S. (2008). Pirate radio in the 1980s: A case study of Thessaloniki 's pirate radio. Unpublished Doctoral Dissertation. Panteion University of Social and Political Sciences.

Tufan, F. (2014). New possibilities provided by social networks to radio broadcasting practices: Radio 2.0. Journal of Media Critiques, Special issue(1), 87-101. https://doi.org/10.17349/jmc114105.

Zelenkauskaite, A., \& Simoes, B. (2015). User interaction profiling across radio stations on Facebook, Twitter, and Google +, Digital and social media- Collective intelligence and Crowds in: Structure, roles, and identity. Proceedings of the Fofty-Eight Hawaii International Conference on System Sciences (HICSS-48), Hawaii, Kauai, 5-8 January 2015, 1657-1666. https://doi.org/10.1109/HICSS.2015.199

Zudrell, M. (2016). Factors affecting branded posts popularity and fan page engagement. Unpublished Bachelor Thesis, Modul University.

\section{Copyright Disclaimer}

Copyright for this article is retained by the author(s), with first publication rights granted to the journal.

This is an open-access article distributed under the terms and conditions of the Creative Commons Attribution license (http://creativecommons.org/licenses/by/3.0/). 